\title{
Un modèle pour le calcul des déformations et des contraintes résiduelles dans les couches d'oxyde lors de chargements thermiques à régime variable
}
A. Touati $\left({ }^{1}\right)$, J.M. Roelandt $\left({ }^{1}\right)$, F. Armanet $\left({ }^{2}\right)$, M. Lambertin( $\left({ }^{1}\right)$ et G. Béranger $\left({ }^{1}\right)$
( $\left.{ }^{1}\right)$ Laboratoire de Génie Mécanique pour les Matériaux et les Structures, URA CNRS 1505, Université de Technologie de Compiègne, BP 649, 60206 Compiègne Cedex, France
$\left({ }^{2}\right)$ Embassy of France, 4101 Reservoir road N.W., 20007, 2176, Washington D.C., U.S.A.

\begin{abstract}
Résumé . - Nous présentons un modèle unidimensionnel de type éléments finis concernant les milieux oxydés dans des conditions de sollicitations thermiques à régime variable. Ce modèle prend en compte la croissance des couches d'oxyde au cours du temps, les relations d'épitaxie et de variations de volume à l'interface métal-oxyde, les comportements visco-plastiques du métal ainsi que la variation des coefficients mécaniques en fonction de la température. Ce modèle permet de déterminer et de suivre par le calcul les variations des contraintes dans l'épaisseur de la couche d'oxyde pendant des cycles de chargement mécanique ou thermique. En particulier, nous avons étudié l'influence de la vitesse de refroidissement sur le niveau des contraintes résiduelles. Les résultats numériques ont fait l'objet d'une confrontation avec l'expérience et paraissent en bonne concordance avec les mesures des contraintes résiduelles effectuées par la diffraction des rayons $\mathrm{X}$ sur le système $\mathrm{Ni} / \mathrm{NiO}$.
\end{abstract}

\begin{abstract}
A one-dimensional model based on finite elements calculation is proposed in order to explain the mechanical behaviour of metal-oxide systems. In spite of a general approach this model has been specially applied to the $\mathrm{NiO} / \mathrm{Ni}$ system. This model takes into account the thickening of the oxide layer, the epitaxial misfit between the oxide scale and the metallic substrate, the volume variations, the visco-plastic deformations of the materials and the effect of temperature on the mechanical coefficients of the system. This model leads to calculate the variation of the residual stresses in the oxide scale and to relate them to thermal cycling conditions or variations of cooling rates. The calculated values are compared to experimental data obtained by X-RD; this comparison demonstrates a good agreement.
\end{abstract}

\section{Introduction.}

Les composants mécaniques, soumis à des chargements thermiques sévères ainsi qu'à l'effet d'un environnement agressif, peuvent subir des modifications structurales et une oxydation superficielle. Le matériau mis en jeu se transforme alors en un composé métal-oxyde. Ces transformations sont accompagnées en général, par l'apparition de concentrations de contraintes, localisées dans la zone adjacente à l'interface métal-oxyde ainsi constitué. Ces contraintes sont dûes [1]:

- d'une part, à la déformation thermo-mécanique localisée principalement à l'interface métal-oxyde dûe à la discontinuité des coefficients mécaniques entre ces deux matériaux [1, 8]; 
- et d'autre part, à certains effets microscopiques de la déformation, telle que l'incompatibilité des réseaux cristallins entre le métal et l'oxyde qui engendre des déformations associées aux variations de volume et à l'épitaxie avec dislocations interfaciales $[2,3,10]$.

Mis à part les travaux de Bernstein [2], les études numériques concernant la modélisation du comportement mécanique de ces matériaux ainsi que le calcul des contraintes dans les couches d'oxyde sont semi-empiriques [3-8]. Toutefois, les travaux expérimentaux sont nombreux, notamment les mesures des contraintes résiduelles utilisant la diffraction des rayons $\mathrm{X}[9,11]$.

Nous présentons dans cette étude, un modèle numérique permettant le calcul des contraintes résiduelles dans les couches d'oxyde sous des chargements thermiques à régime variable.

\section{Modèle.}

Les caractéristiques principales du modèle sont :

a) Le modèle est unidimensionnel, la variation des déformations et des contraintes est étudiée uniquement dans l'épaisseur d'oxyde (direction $o x_{2}$ ) (Fig. 1). Les déformations dans le plan de l'interface métal-oxyde sont ainsi supposées identiques ;
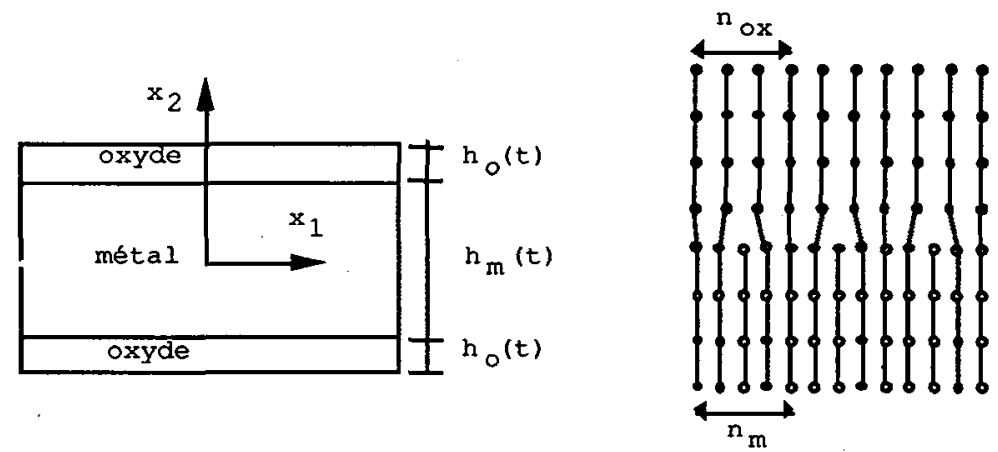

Fig. 1. - Description du modèle avec prise en compte de l'épitaxie.

b) la structure métal-oxyde (Fig. 1) est considérée comme un matériau constitué de deux couches externes d'oxyde, dont l'épaisseur $h_{0}(t)$ évolue avec le temps et la température suivant une loi parabolique (1) et d'une matrice métallique d'épaisseur $h_{\mathrm{m}}$ supposée constante (cette restriction aurait peu d'influence sur les valeurs obtenues dans l'oxyde).

$$
h_{0}(t, T)=K_{0} \cdot \exp \left(-\frac{Q}{k T}\right) t^{0,5}=K_{\mathrm{p}}(T) t^{0,5}
$$

( $K_{0}$ est une constante, $Q$ est l'énergie d'activation, $k$ est la constante de Boltzman, et $T$ est la température) ; 
c) à l'interface métal-oxyde il y a superposition des déformations de variation de volume et d'épitaxie. Leur expression découle d'un transfert micro-macroscopique de la déformation associée à chacun de ces phénomènes $[2,6,7,10]$;

d) le comportement du métal ainsi que de l'oxyde est considéré comme élasto-viscoplastique, tenant compte de l'effet de fluages primaire et secondaire [2]. L'hypothèse des contraintes planes est prise en compte $\sigma_{2}\left(x_{2}\right)=0$;

e) les déformations sont planes. Les déformations totales exprimées dans le métal et dans l'oxyde sont données par la relation :

$$
\varepsilon_{i}=\varepsilon_{i}^{\mathrm{e}}+\varepsilon_{i}^{\mathrm{th}}+\varepsilon_{i}^{\mathrm{vp}}+\varepsilon_{i}^{\mathrm{ox}} i=1,2 \quad\left(\varepsilon_{i}^{\mathrm{ox}}=0 \text { dans le métal }\right)
$$

Les différentes contributions aux déformations sont :

- les déformations thermo-mécaniques classiques :

$\varepsilon_{1}^{\mathrm{el}}, \varepsilon_{2}^{\mathrm{el}}$ sont les déformations élastiques; $\sigma_{1}=\frac{E}{1-\nu^{2}}\left(\varepsilon_{1}^{\mathrm{el}}+\nu \varepsilon_{2}^{\mathrm{cl}}\right)$

$\varepsilon_{\mathrm{i}}^{\mathrm{vp}}$ sont les déformations visco-plastiques

$\varepsilon_{\mathrm{i}}^{\text {th }}$ sont les déformations thermiques : $\varepsilon_{\mathrm{i}}^{\text {th }}=\alpha(T)\left(T-T_{0}\right) i=1,2$

L'évolution des déformations visco-plastiques des matériaux, en fonction des contraintes et du temps est décrite par les relations suivantes :

$$
\dot{\varepsilon}_{\mathrm{e}}^{\mathrm{vp}}=A\left(\sigma_{\mathrm{e}}\right)^{m} t^{p}+B\left(\sigma_{\mathrm{e}}\right)^{n}
$$

où : $\dot{\varepsilon}_{\mathrm{e}}^{\mathrm{vp}}=\left(\frac{2}{3}\left(2 \dot{\varepsilon}_{1}^{\mathrm{vp}}+\dot{\varepsilon}_{2}^{\mathrm{vp}}\right)\right)^{\frac{\mathrm{l}}{2}}$ (taux de déformation visco-plastique équivalente)

$\sigma_{\mathrm{e}}=\sigma_{1}\left(x_{2}\right)$ (contrainte équivalente de Von-Mises)

$2 \varepsilon_{1}^{\mathrm{vp}}+\varepsilon_{2}^{\mathrm{vp}}=0$ (incompressibilité des déformations viscoplastiques)

- les déformations d'oxydation :

Celles-ci regroupent :

Les déformations de variation de volume couplées avec l'effet de la température [6-7] :

$$
\varepsilon_{1}^{\mathrm{vv}}(\mathrm{T})=\varepsilon_{2}^{\mathrm{vv}}(\mathrm{T})=\left(\left(P B R \cdot \frac{1+\alpha_{0}(T) \cdot\left(T-T_{0}\right)}{1+\alpha_{\mathrm{m}}(T) \cdot\left(T-T_{0}\right)}\right)^{\frac{1}{3}}-1\right)
$$

où $\alpha_{0}$ et $\alpha_{\mathrm{m}}$ sont les coefficients de dilatation de l'oxyde, du métal et $P B R$ est le coefficient de Pilling et Bedworth.

- La déformation d'épitaxie (couplée avec la déformation de variation de volume) :

Le phénomène d'épitaxie engendre des déformations locales, dans la zone adjacente à l'interface métal-oxyde, qui s'opposent aux déformations dites de variation de volume. Ces déformations sont principalement à l'origine des dislocations interfaciales [10], (Fig. 2). Lexpression des déformations d'oxydation $\varepsilon_{1}^{\text {ox }}, \varepsilon_{2}^{\text {ox }}$ (épitaxie couplée à la variation de volume) peut être exprimée de manière approchée par la relation suivante [6-7] :

$$
\varepsilon_{i}^{\mathrm{ox}}=\left(1-\frac{n_{\mathrm{m}}}{n_{0}}\right) \cdot \varepsilon_{i}^{\mathrm{vv}}
$$


où $n_{\mathrm{m}}$ et $n_{0}$ sont les nombres de plans cristallins du métal et de l'oxyde entre deux dislocations consécutives.

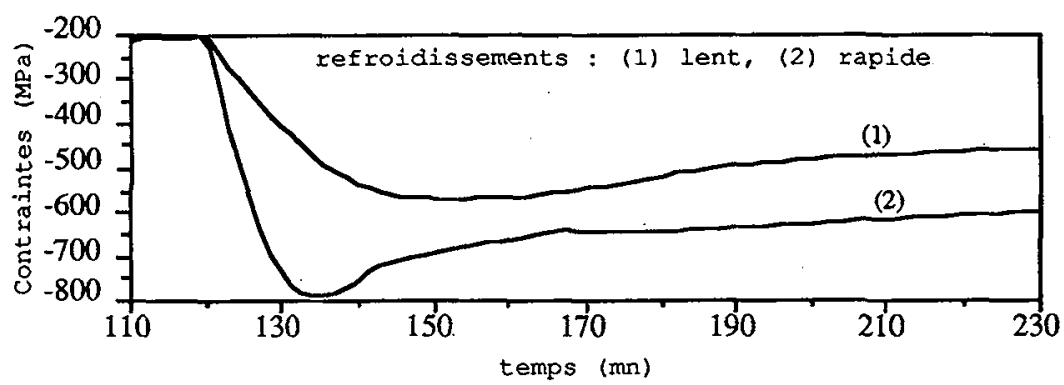

Fig. 2. - Contraintes dans la couche d'oxyde pendant le refroidissement et contraintes résiduelles après retour à la température ambiante.

\section{Modèle numérique.}

Nous utilisons un processus numérique incrémental, suivant l'algorithme de CrankNicholson, pour intégrer les lois qui régissent le modèle.

Pendant un acroissement $\Delta t_{i}$ du temps, l'accroissement $\Delta h_{i}$ de la couche d'oxyde est calculé par intégration de la loi (1). L'épaisseur $\Delta h_{1}$ du métal est considérée comme constante au cours du temps : $\Delta h_{1}=h_{\mathrm{m}} / 2$. Les dégrés de liberté sont les déformations $\left(\varepsilon_{1}^{i}, \varepsilon_{2}^{i}\right)$ et les contraintes $\left(\sigma_{1}^{i}\right)$ dans les différentes couches d'indice $i$ et d'épaisseur $\Delta h_{i}$. L'équilibre dans le matériau métal/oxyde est réalisé à l'instant $t_{i}$ lorsque :

$$
\sum_{j=1}^{i} \int_{h_{j}}^{h_{j+1}} \sigma_{1}^{i} \mathrm{~d} x_{2}=\sum_{j=1}^{i} \Delta h_{j} \sigma_{1}^{i}=0
$$

Les relations $(2,3,4,5,6)$ conduisent à l'expression de l'équilibre sous la forme incrémentale suivante :

$$
\begin{gathered}
\sum_{j=1}^{i} \Delta h_{j} \frac{E_{j}}{1-\nu_{j}^{2}}\left(\Delta \varepsilon_{1}^{j}+\nu_{j} \Delta \varepsilon_{2}^{j}\right)=\sum_{j=1}^{i} \Delta h_{j} \frac{E_{j}}{1-\nu_{j}^{2}}\left(\left(\Delta \varepsilon_{1}^{\mathrm{th} j}+\Delta \varepsilon_{1}^{\mathrm{vp} j}\right)+\right. \\
\nu_{j}\left(\Delta \varepsilon_{2}^{\mathrm{th} j}+\Delta \varepsilon_{2}^{\mathrm{vp} j}\right)+\Delta h_{2} \frac{E_{2}}{1-\nu_{2}^{2}}\left(\left(\Delta \varepsilon_{1}^{\mathrm{ox}}+2 \Delta \varepsilon_{2}^{\mathrm{ox}}\right)\right)
\end{gathered}
$$

Les déformations d'oxydation ne sont prises en compte que dans la première couche d'oxyde. Nous faisons l'hypothèse que les déformations d'une couche d'oxyde sont égales au cumul des déformations du métal depuis l'instant de sa création [2] :

$$
\varepsilon_{k}^{i}\left(t_{j}\right)=\varepsilon_{k}^{1}\left(t_{j}\right)-\varepsilon_{k}^{1}\left(t_{i}-1\right) \quad k=1,2
$$

La prise en compte de la relation (9) dans (8) conduit à la résolution d'un système non linéaire à deux inconnues $\left(\Delta \varepsilon_{1}^{1}, \Delta \varepsilon_{2}^{1}\right)$. Les valeurs des contraintes sont calculées à partir des relations $(4,5)$. 


\section{Exemples et résultats.}

Le premier exemple numérique est relatif à l'étude des effets d'un maintien à une température constante de l'échantillon pendant un temps $t_{\mathrm{m}}$ (afin d'accéder à une épaisseur donnée de la couche d'oxyde) puis à un refroidissement de l'échantillon suivant différentes vitesses.

Les données géométriques et des sollicitations thermiques sont les suivantes :

- la température d'oxydation est de $1200 \mathrm{~K}$;

- $h_{\mathrm{m}}=3 \mathrm{~mm}, t_{\mathrm{m}}=2 \mathrm{~h}, \dot{T}=45 \mathrm{~K} / \mathrm{mn}$ (refroidissement rapide) ou $\dot{T}=15 \mathrm{~K} / \mathrm{mn}$ (refroidissement lent);

- les épaisseurs d'oxyde après refroidissement sont de $0.00364 \mathrm{~mm}$ dans le cas du refroidissement rapide et de $0.00372 \mathrm{~mm}$ dans le cas lent.

Les données matérielles ou physiques sont [2-6-7] :

Modules de Young (métal et oxyde) : $E(T)=a(1-\exp (b T))+c$

$a=20400 \mathrm{MPa}, b=1,45 \times 10^{-3} /{ }^{\circ} \mathrm{C}, c=200600 \mathrm{MPa}$ (dans le métal),

$a=33400 \mathrm{MPa}, b=1,45 \times 10^{-3} /{ }^{\circ} \mathrm{C}, c=243000 \mathrm{MPa}$ (dans l'oxyde).

Coefficient de Poisson : $\nu=0,3$ (métal et oxyde).

Coefficients de dilatation : $\alpha(T)=\mathrm{d} T+e$

$d=4,47 \times 10^{-9} \circ / \mathrm{C}^{2}, e=1,81 \times 10^{-5} /{ }^{\circ} \mathrm{C}$ (dans le métal),

$d=3 \times 10^{-9} /{ }^{\circ} \mathrm{C}^{2}, e=1,5 \times 10^{-5} /{ }^{\circ} \mathrm{C}$ (dans l'oxyde).

Epitaxie et variation de volume :

$P B R\left(20^{\circ} \mathrm{C}\right)=1,65, K_{\mathrm{p}}(T)=0,353 \exp (-10430 / T) \mathrm{mm} \mathrm{s}^{-1 / 2}$,

$1-\frac{n_{\mathrm{m}}}{n_{0}}=0,11, a_{\mathrm{m}}=3,524 \AA, a_{0}=4,1769 \AA$.

Lois visco-plastiques : $\dot{\varepsilon}_{\mathrm{e}}^{\mathrm{vp}}=\mathrm{A}\left(\sigma_{\mathrm{e}}\right)^{m} \mathrm{t}^{p}+\mathrm{B}\left(\sigma_{\mathrm{e}}\right)^{n}$

$A=1,028 \times 10^{-5}, m=2,392, p=-2 / 3, B=3,16 \times 10^{-9}, n=4,6$ (dans le métal),

$A=0, B=5,343 \times 10^{-3}, n=3,06$ (dans l'oxyde).

\section{Résultats :}

Le refroidissement est accompagné d'une élévation du niveau des contraintes dans la couche d'oxyde, suivie d'une période de relaxation après retour à la température ambiante du chargement thermique. Un refroidissement lent favorise la relaxation des contraintes ; elles est de l'ordre de $8 \%$ supérieure dans le cas lent par rapport au cas rapide, (Fig. 2).

\section{Comparaison calculs / expériences.}

La comparaison des résultats numériques avec l'expérience a consisté à procéder à des simulations sur différents échantillons. On a considéré différents temps de maintien à température constante $(3,7,11,16,23,35,54 \mathrm{~h})$ conduisant à différentes épaisseurs totales de la couche d'oxyde $(4,7,9,11,13,19$ et $20 \mathrm{~mm})$, suivis de refroidissements. Les données matérielles et physiques, la température d'oxydation, l'épaisseur initiale sont les mêmes que précédemment. Les résultats numériques sont comparés, après stabilisation de l'évolution des contraintes résiduelles, avec les mesures par diffraction des rayons X, (Fig. 3). 


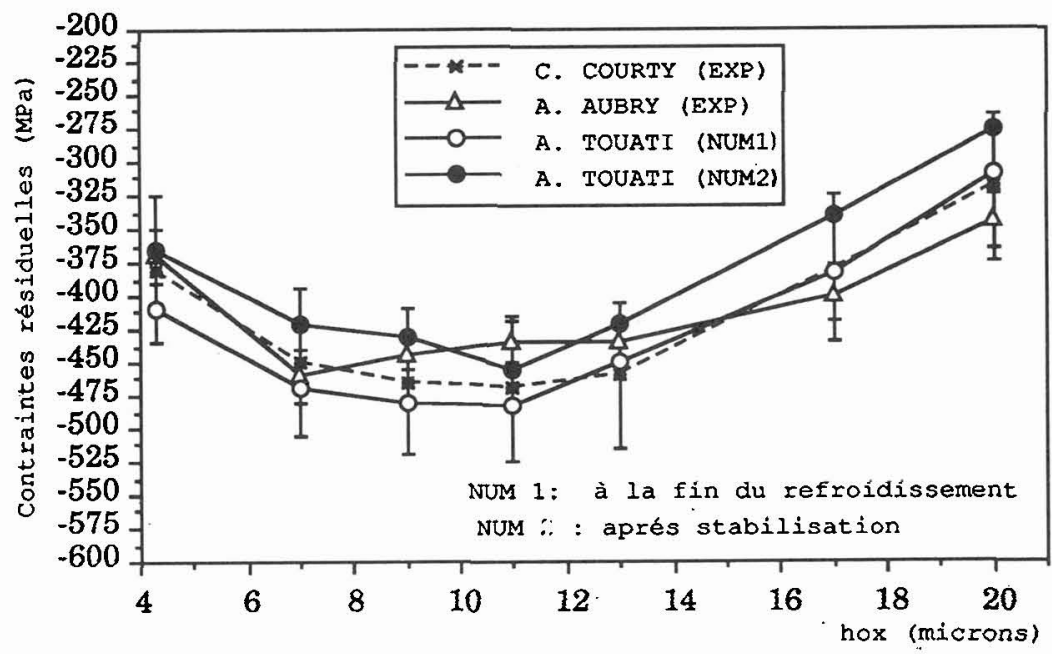

Fig. 3. - Contraintes dans le système NiO pour différentes épaisseurs d'oxyde (résultats numériques - mesures $\mathbf{r X}$ ).

\section{Conclusion.}

Le modèle proposé ainsi que le logiciel associé, permettent de suivre par le calcul l'évolution des contraintes dans la couche d'oxyde pendant une phase d'oxydation ou d'élaboration d'un revêtement par pré-oxydation volontaire du matériau ainsi que lors de la phase de refroidissement qui s'ensuit (Fig. 2). Le niveau des contraintes résiduelles obtenues par le calcul a été comparé avec les valeurs expérimentales dans le cas du système $\mathrm{Ni} / \mathrm{NiO}$. Les résultats numériques, obtenus pour différentes vitesses de refroidissement, sont en bonne concordance avec les résultats expérimentaux (Fig. 3).

Ce modèle, qui nécessite certaines améliorations, donne une bonne approximation du comportement des couches d'oxyde formées sur un matériau soumis à une atmosphère oxydante à haute température.

\section{Bibliographie}

[1] Béranger G., Huntz A.M., Pieraggi B., Ecole du C.N.R.S. (Piau-Engaly, France, 1985).

[2] BERNSTEIN H.L., Metall. Trans. 18A (1987) 975.

[3] Evans H.E., Corros. Sci. 23 (5) (1983) 495.

[4] Fromhold A.T., Stress effects and the oxidation of metals, J.V. Cathcart Ed. (The metallurgical society of AIME, 1974).

[5] SRolovitz D.J., Ramanarayanan T.A., Oxid. Met. 22 (1984) 314.

[6] Touati A., Thèse de Doctorat, Université de Technologie de Compiègne (1991). 
[7] Touati A., Roelandt J.M., Armanet F,, BÉranger G., Int. Conf. on Advanced Materials (ICAM 1991, Strasbourg, 1991).

[8] Oxx G.D., Prod. Eng. 29 (1958) 61.

[9] CoURTY C., Thèse de Doctorat, UTC (1988).

[10] PIERAGgi B., RAPP R.A., TMS ASM, Norm Peterson Symposium on High Temperature Corrosion, Dayananda, King, Rothman Eds. (TMS-AIME, Werrendale, PA, 1987).

[11] Aubry A., Armanet F., Béranger G., Lebrun J.L., Maeder G., Acta. Metall. 36 (1988) 2779. 